\title{
Sekundärcarotinoide in der Grünalge Sphaeroplea
}

\author{
HANS KLeINIG $^{+}$ \\ Botanisches Institut der Universität Heidelberg \\ (Z. Naturforschg. 22 b, 977-979 [1967] ; eingegangen am 16. März 1967)
}

\begin{abstract}
The secondary carotenoids of the zygospores of the green alga Sphaeroplea cambrica have been investigated. The zygospores contain 3-hydroxy-4-oxo- $\beta$-carotene (hydroxyechinenone), 3,3'-dihydroxy-4-oxo- $\beta$-carotene (adonixanthin), 3-hydroxy-4,4'-dioxo- $\beta$-carotene (adonirubin) and 3,3'-dihydroxy-4,4'-dioxo- $\beta$-carotene (astaxanthin) esterified with myristic, lauric and caprinic acid and 4 -oxo- $\beta$-carotene (echinenone) and $4,4^{\prime}$-dioxo- $\beta$-carotene (canthaxanthin).
\end{abstract}

Die Gattung Spheroplea wird im Algensystem zu den Ulotrichales gestellt. Sie ist gekennzeichnet durch mehrkernige Zellen und Oogamie. Es ist bekannt, daß sich diese Alge, die oft in Massen auftritt, manchmal rot anfärbt. Auch einige andere Grünalgen nehmen unter bestimmten Bedingungen

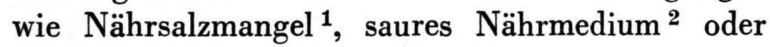
starker Beleuchtung ${ }^{3}$ eine rote Farbe an. Als Farbstoffe wurden die Carotinoide Astaxanthin ${ }^{4}$, Echinenon ${ }^{5}$, Canthaxanthin ${ }^{6}$, Hydroxyechinenon, Adonixanthin und Adonirubin ${ }^{7}$ in den Algen gefunden. Soweit diese Pigmente Hydroxylgruppen besitzen, sind sie mit höheren Fettsäuren verestert. Diese Carotinoide unterscheiden sich sowohl in ihrer Struktur als auch in ihrer Bildungsweise von den übrigen in den Algen normalerweise vorhandenen Carotinoiden ( $\alpha$-Carotin, $\beta$-Carotin, Zeaxanthin, Lutein, Antheraxanthin, Luteinepoxid, Violaxanthin, Neoxanthin) und werden als Sekundärcarotinoide bezeichnet. Sekundärcarotinoide wurden bisher in Haematococcus ${ }^{4}$, Chlorella, Scenedesmus, Ankistrodesmus, Hydrodictyon 1, 2, 8, Acetabularia $^{3,7}$, Spongiochloris ${ }^{8 a}$ u. a. nachgewiesen. In Trentepohlia kann $\beta$-Carotin als Sekundärpigment auftreten ${ }^{9}$.

1952 wurde auf der Schwäbischen Alb ein rotes Geflecht von Sphaeroplea cambrica Fritsch gefunden ${ }^{10}$, die auf Grund der sehr stark strukturierten

* Anschrift: Botanisches Institut der Universität Freiburg, Schänzlestraße.

1 G. Dersch, Flora [Jena] 149, 566 [1960].

2 E. Kessler u. F. C. Czygan, Ber. dtsch. bot. Ges. 78, 342 [1965].

3 H. Kleinig, Ber. dtsch. bot. Ges. 79, 126 [1966].

4 J. Tischer, Hoppe-Seyler's Z. physiol. Chem. 281, 143 [1944].

5 F. C. Czygan, Z. Naturforschg. 21 b, 197 [1966].

6 F. C. Czygan, Experientia [Basel] 20, 573 [1964].

7 H. Kueinig u. K. EGGER, Phytochem. 6, 611 [1967].
Wand ihrer Zygosporen zu einer neuen Varietät Sphaeroplea cambrica FRITSCH var. crassisepta RIETH erhoben worden ist ${ }^{11}$.

Untersucht wurde dieses Typusmaterial, das fast ausschließlich stark rot gefärbte Zygosporen in den Zellen enthielt *.

\section{Untersuchungen und Ergebnisse}

1. Die Farbstoffe: Es zeigte sich, daß auch hier rote z. T. veresterte Carotinoide vorlagen. Zur Identifizierung wurde das Material mit Aceton und Petroläther extrahiert und über Kieselgel chromatographiert. Die einzelnen Fraktionen wurden mit 10-proz. Natriumalkoholat verseift und nach Ansäuern mit Essigsäure in Petroläther überführt. Bei dieser Verseifung werden Hydroxylgruppen in a-Stellung zu Ketogruppen selbst zu Ketogruppen oxydiert. Ketogruppen wurden durch Reduktion mit $\mathrm{NaBH}_{4}$ in Äthanol, Hydroxylgruppen durch Acetylierung mit Essigsäureanhydrid in Pyridin, allylständige Hydroxyle außerdem durch Äthylierung in $0,01 \mathrm{~N}$ äthanolischer $\mathrm{HCl}^{12}$ nachgewiesen. Als chromatographische Systeme dienten neben KieselgelDünnschichten mit Paraffinöl imprägnierte Cellulose-Dünnschichten für die Carotinoidester und mit ungesättigten Triglyceriden imprägnierte Cellulose-

8 E. Kessler, W. Langner, J. Ludewig u. H. Wichmann, Studies on Microalgae and Photosynthetic Bacteria 1963, 7.

8a R. McLean, Physiol. Plant. 20, 41 [1967].

9 F. C. Czygan u. K. Kalb, Z. Pflanzenphysiol. 55, 59 [1966].

10 Тн. Butterfass, Jh. Ver. vaterl. Naturk. Württ. 108, 142 [1953].

11 A. Rieth, Flora [Jena] 140, 130 [1953].

* Herr Doz. Dr. Th. Butrerfass, Max-Planck-Institut Rosenhof, Ladenburg, stellte das Material zur Verfügung, wofür ich ihm sehr danken möchte.

12 E. C. Grob u. R. P. Prlugshaupt, Helv. chim. Acta 45, 1592 [1962]. 
Dünnschichten für die freien Pigmente ${ }^{13}$ 14. Die Absorptionsspektren wurden mit einem registrierenden Spektralphotometer ZEISS RPQ 20 aufgenommen. Als Vergleichssubstanzen wurden die aus den Petalen von Adonis $^{\mathbf{1 5}}$ und aus Acetabularia ${ }^{7}$ bekannten Hydroxyechinenon (3-Hydroxy-4-keto- $\beta$ carotin), Adonixanthin (3.3'-Dihydroxy-4-keto- $\beta$ carotin), Adonirubin (3-Hydroxy-4.4'-diketo- $\beta$ carotin) und Astaxanthin (3.3'-Dihydroxy-4.4' ${ }^{\prime}$-diketo- $\beta$-carotin) bzw. deren Oxydationsprodukte und authentisches Echinenon (4-Keto- $\beta$-carotin) und Canthaxanthin (4.4' $4^{\prime}$-Diketo- $\beta$-carotin) verwendet ${ }^{* *}$.

Die Analysen ergaben, daß neben Astaxanthinestern als Hauptkomponenten noch die Ester von drei weiteren Ketohydroxycarotinoiden Hydroxyechinenon, Adonixanthin und Adonirubin und die reinen Ketocarotinoide Echinenon und Canthaxanthin in Sphaeroplea enthalten sind. Chlorophylle und Primärcarotinoide waren in den Zygosporen nicht nachzuweisen. Die spektralen Daten und die prozentuale Verteilung sind aus der Tab. 1 zu entnehmen.

\begin{tabular}{|c|c|c|c|}
\hline Pigment & $\begin{array}{c}\text { rel. } \\
\text { Menge } \\
{[\%]}\end{array}$ & $\begin{array}{l}\text { Absorptions- } \\
\text { maxima der } \\
\text { nativen } \\
\text { Pigmente } \\
\text { in Äthanol }\end{array}$ & $\begin{array}{l}\text { Absorptions- } \\
\text { maxima der } \\
\text { reduzierten } \\
\text { Pigmente }\end{array}$ \\
\hline $\begin{array}{l}\text { Echinenon } \\
\quad(4 \text {-Keto- } \beta \text {-carotin })\end{array}$ & 2 & 466 & 448,474 \\
\hline $\begin{array}{l}\text { Hydroxyechinenon } \\
\text { (3-Hydroxy-4-keto- } \\
\beta \text {-carotin) }\end{array}$ & 2 & 466 & 448,474 \\
\hline $\begin{array}{l}\text { Adonixanthin } \\
\left(3.3^{\prime} \text {-Dihydroxy-4- }\right. \\
\text { keto- } \beta \text {-carotin) }\end{array}$ & 21 & 465 & 448,475 \\
\hline $\begin{array}{l}\text { Canthaxanthin } \\
\left(4.4^{\prime} \text {-Diketo- }\right. \\
\beta \text {-carotin })\end{array}$ & 3 & 478 & 448,475 \\
\hline $\begin{array}{l}\text { Adonirubin } \\
\text { (3-Hydroxy-4.4'- } \\
\text { diketo- } \beta \text {-carotin) }\end{array}$ & 4 & 478 & 448,475 \\
\hline $\begin{array}{l}\text { Astaxanthin } \\
\left(3.3^{\prime}-\text { Dihydroxy-4.4'- }\right. \\
\text { diketo- } \beta \text {-carotin })\end{array}$ & 68 & 478 & 448,475 \\
\hline
\end{tabular}

Tab. 1. Spektrale Daten und prozentuale Verteilung (s. Text).

Adonixanthin:

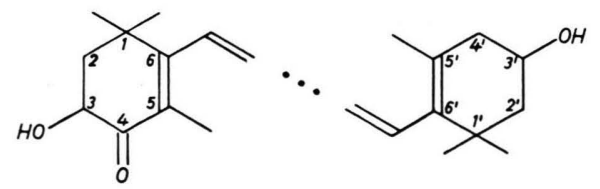

13 K. EGGER, Chromatogr. Sympos. II, 75 Brüssel 1962.

14 K. EGGER, Planta 58, 664 [1962].

15 K. EGGER, Phytochem. 4, 609 [1965].
2. Die Fettsäurekomponenten: Die hydroxylhaltigen Carotinoide in Sphaeroplea sind mit höheren Fettsäuren verestert. Jede auf dem Adsorptionschromatogramm einheitliche Fraktion spaltet auf dem Verteilungschromatogramm (imprägnierte Celluloseschicht) in Banden auf, die auf Veresterung mit mehreren Säuren weisen und für Sekundärcarotinoide typisch sind. Zur Bestimmung dieser Fettsäuren kann eine Veresterung der verseiften Pigmente mit Carbonsäurechloriden und anschließendem Vergleich auf dem Chromatogramm nicht durchgeführt werden, da die Pigmente nach der Verseifung in der Ketoform vorliegen. Eine direkte Analyse der freien Säuren kann durch begleitende Lipide verfälscht werden und ergibt keine exakte Zuordnung zu den einzelnen Pigmenten.

Die Petalen von Adonis annua enthalten ebenfalls Fettsäureester von Astaxanthin, Adonirubin, Adonixanthin und Hydroxyechinenon ${ }^{15}$. Hier konnten die Säuren auf andere Weise identifiziert werden ${ }^{\mathbf{1 6}}$ : an der Veresterung sind Palmitin-, Myristin-, Laurinund Caprinsäure und eine ungesättigte Säure beteiligt. Somit liegen definierte Vergleichssubstanzen vor. Der Vergleich der unbekannten Ester aus Sphaeroplea mit den bekannten Estern aus Adonis in verschiedenen chromatographischen Systemen zeigte, daß in den Carotinoidestern von Sphaeroplea die Myristinsäure Hauptkomponente, Laurin- und Caprinsäure Nebenkomponenten sind.

\section{Diskussion}

Einige Grünalgen (Haematococcus ${ }^{4}$, Chlorella, Ankistrodesmus, Scenedesmus, Hydrodictyon 1, 2, 8 und Acetabularia ${ }^{3,7}$ ) bilden unter bestimmten Umweltsbedingungen Sekundärcarotinoide. Meist werden dabei Chlorophylle und Primärcarotinoide abgebaut. Der Prozeß ist reversibel. Im allgemeinen ist Nährsalzmangel der auslösende Faktor ${ }^{1}$, aber auch ein niederer $p_{\mathrm{H}^{-}}$Wert der Nährlösung bei Chlorella zofingiensis ${ }^{2}$ und starke Beleuchtung bei Acetabularia $^{3}$ können zu dieser merkwürdigen Pigmentänderung führen. Vermutlich bewirken alle genannten auslösenden Faktoren die gleiche Umorientierung des Stoffwechsels in den betreffenden Algen. Über den Mechanismus dieses Vorganges selbst und über eine eventuelle Bedeutung lassen sich bisher

\footnotetext{
** Herrn Prof. Dr. Weedon, London, danke ich für die Uberlassung der beiden synthetischen Substanzen.

16 K. EgGer u. H. Kleinig, Phytochem. 6, 437 [1967].
} 
keine sicheren Aussagen machen. Die zahlreichen neu synthetisierten Carotinoide können auf einen Grundtyp zurückgeführt werden, der in fast allen möglichen Variationen erscheint: 3-Hydroxy-4-keto$\beta$-carotine, wobei mindestens eine Ketogruppe in Konjugation zum Doppelbindungssystem stehen muß. Die Hydroxyle sind mit verschiedenen höheren Fettsäuren verestert. Aus der Tab. 1 wird ersichtlich, daß eine letzte noch mögliche Variation, das $3^{\prime}$-Hydroxy-4-keto- $\beta$-carotin, bisher nicht als Sekundärcarotinoid gefunden worden ist. Dieses Pigment ist jedoch schon als Primärcarotinoid aus Cyanophyeen bekannt ${ }^{17}$. Die höchstoxydierte Form, das Astaxanthin, ist in allen Sekundärcarotinoidführenden Algen Hauptfarbstoff. Die übrigen Pigmente können in den einzelnen Algen z. T. fehlen.

Die Sekundärcarotinoide von Sphaeroplea cambrica fügen sich in dieses Schema ein. Bemerkenswert ist die Vielzahl der Verbindungen und die hohe Konzentration an Adonixanthinestern.

Ein anderer Modus der Sekurdärcarotinoid-Bildung ist in Trentepohlia gefunden worden ${ }^{9}$. In dieser Alge kann $\beta$-Carotin als Sekundärfarbstoff erscheinen. Ketocarotinoide sind nicht vorhanden.

Die Deutsche Forschungsgemeinschaft hat die Untersuchungen durch Sachmittel gefördert.

17 S. Hertzberg u. S. Lianen Jensen, Phytochem. 5, 557 [1966]. 